\title{
DIRECTIONAL PROPERTIES OF SETS DEFINABLE IN O-MINIMAL STRUCTURES
}

\author{
SATOSHI KOIKE, TA LÊ LOI, LAURENTIU PAUNESCU AND MASAHIRO SHIOTA
}

\begin{abstract}
In 9 it was introduced the notion of direction set for a subset of $\mathbb{R}^{n}$, and it was shown that the dimension of the common direction set of two subanalytic subsets, called directional dimension, is preserved by a bi-Lipschitz homeomorphism, provided that their images are also subanalytic. In this paper we give a generalisation of the above result to sets definable in an o-minimal structure on an arbitrary real closed field. More precisely, we first prove our main theorem and discuss in detail directional properties in the case of an Archimedean real closed field, and in $\S 7$ we give a proof in the case of a general real closed field. In addition, related to our main result, we show the existence of special polyhedra in some Euclidean space, illustrating that the bi-Lipschitz equivalence does not always imply the existence of a definable one.
\end{abstract}

\section{INTRODUCTION}

We first recall the notions of direction set and real tangent cone in $\mathbb{R}^{n}$.

Definition 1.1. Let $A$ be a set-germ at $0 \in \mathbb{R}^{n}$ such that $0 \in \bar{A}$. We define the direction set $D(A)$ of $A$ at $0 \in \mathbb{R}^{n}$ by

$$
D(A):=\left\{a \in S^{n-1} \mid \exists\left\{x_{i}\right\} \subset A \backslash\{0\}, x_{i} \rightarrow 0 \in \mathbb{R}^{n} \text { s.t. } \frac{x_{i}}{\left\|x_{i}\right\|} \rightarrow a, i \rightarrow \infty\right\} .
$$

Here $S^{n-1}$ denotes the unit sphere centred at $0 \in \mathbb{R}^{n}$.

We denote by $L D(A)$ a half-cone of $D(A)$ with the origin $0 \in \mathbb{R}^{n}$ as the vertex:

$$
L D(A):=\left\{t a \in \mathbb{R}^{n} \mid a \in D(A), t \geq 0\right\},
$$

and call it the real tangent cone of $A$ at $0 \in \mathbb{R}^{n}$.

Let us examine an example.

Example 1.2. Let $h: \mathbb{R}^{3} \rightarrow \mathbb{R}^{3}$ be a semialgebraic homeomorphism defined by $h(x, y, z)=$ $\left(x, y, z^{3}\right)$, and let $V=\left\{(x, y, z) \in \mathbb{R}^{3}: x^{2}+y^{2}-z^{6}=0\right\}$. Then $V$ and $h(V)=\{(x, y, z) \in$ $\left.\mathbb{R}^{3}: x^{2}+y^{2}-z^{2}=0\right\}$ are algebraic sets. It is easy to see that $\operatorname{dim} D(A)=0$ and $\operatorname{dim} D(h(A))=1$. Therefore the dimension of direction sets is not a homeomorphic invariant.

Date: 9-2-2010.

1991 Mathematics Subject Classification. Primary 14P15, 32B20 Secondary 14P10, 57R45.

Key words and phrases. direction set, o-minimal structure, bi-Lipschitz homeomorphism.

This research is partially supported by the Grant-in-Aid for Scientific Research (No. 20540075) of Ministry of Education, Science and Culture of Japan, and HEM 21 Invitation Fellowship Programs for Research in Hyogo. 
We next investigate whether the dimension of direction sets is a Lipschitz invariant. There are many singular examples of bi-Lipschitz homeomorphisms. In [9] it was given an example of a "quick spiral bi-Lipschitz homeomorphism" and of a "zigzag bi-Lipschitz homeomorphism". Here we give a different one.

Example 1.3. (Oscillation). Let $h:\left(\mathbb{R}^{2}, 0\right) \rightarrow\left(\mathbb{R}^{2}, 0\right)$ be a mapping defined by $h(x, y)=$ $(x, y+f(x))$, where $f(x)=x \sin (\ln |x|)$, and let $A=\mathbb{R} \times 0$. Then we can see that $h$ is a bi-Lipschitz homeomorphism and $h(A)$ is the graph of $f$. In addition, we have $\operatorname{dim} D(A)=0$ and $\operatorname{dim}(D(h(A)))=1$. Consequently the dimension of direction sets is not a bi-Lipschitz invariant either.

Note that in the above example $h(A)$ is not a subanalytic set. Therefore we may ask whether the dimension of direction sets is a bi-Lipschitz invariant in the case when the image $h(A)$ is also subanalytic. In fact, the following result is shown in [9].

Theorem 1.4. (Main Theorem in [9]) Let $A, B \subset \mathbb{R}^{n}$ be subanalytic set-germs at $0 \in \mathbb{R}^{n}$ such that $0 \in \bar{A} \cap \bar{B}$, and let $h:\left(\mathbb{R}^{n}, 0\right) \rightarrow\left(\mathbb{R}^{n}, 0\right)$ be a bi-Lipschitz homeomorphism. Suppose that $h(A), h(B)$ are also subanalytic. Then we have

$$
\operatorname{dim}(D(h(A)) \cap D(h(B)))=\operatorname{dim}(D(A) \cap D(B)) .
$$

See H. Hironaka [6] for subanalyticity.

A subfield of $\mathbb{R}$ is called Archimedean. This terminology comes from the fact that an ordered field $R$ is isomorphic to a subfield of $\mathbb{R}$ if and only if for any positive elements $a$ and $b$ of $R$ there exists a natural number $n$ such that $a n>b$. An ordered field $R$ is called real closed if its complexification $R[t] /\left(1+t^{2}\right) R[t]$ is algebraically closed. The field of real numbers $\mathbb{R}$ is an Archimedean real closed field. Another well-known example of Archimedean real closed field is the field of real algebraic numbers. In this paper we give a generalization of Theorem 1.4 to the case of sets definable in an arbitrary o-minimal structure on an arbitrary real closed field (Theorem 7.1). We first show the main theorem for the case of an Archimedean real closed field. Namely, we show the following:

Theorem 1.5. Let $R$ be an Archimedean real closed field, and let $A, B$ be definable setgerms at 0 in $R^{n}$ in an o-minimal structure on $R$ such that $0 \in \bar{A} \cap \bar{B}$. Let $h:\left(R^{n}, 0\right) \rightarrow$ $\left(R^{n}, 0\right)$ be a bi-Lipschitz homeomorphism. Suppose that $h(A), h(B)$ are also definable. Then we have

$$
\operatorname{dim}(D(h(A)) \cap D(h(B)))=\operatorname{dim}(D(A) \cap D(B)) .
$$

See the next section for the definition of a definable set and of the direction set $D(A)$ of a set $A$ in $R^{n}$.

Theorem 1.4 was shown using essentially the following ingredients:

(1) Sea-tangle properties;

(2) Sequence selection properties;

(3) Volume arguments.

In $\S 2$ we describe the notion of o-minimal structure and point out some of its properties. We give in $\S 3$ an important example concerning the relationship between bi-Lipschitz 
equivalence and definable equivalence. After this we introduce an adapted notion of sea-tangle neighbourhood, using ordered definable functions and describe several of its properties in $\S 4$. In $\S 5$ we discuss sequence selection properties, and we give the proof of our main theorem (Theorem 1.5) using volume arguments in $\S 6$. In $\S \S 4-6$ we develop the arguments in any o-minimal structure on any Archimedean real closed field. In $\S 7$ we generalise the main theorem to any real closed field and give a proof. This proof is rather resembling proofs in Logic Theory. Essentially, this shows that in our proof of the main theorem we do not use all special properties of the real number field (e.g. local compactness).

\section{O-MINIMAL STRUCTURE}

Throughout this paper, except $\S 7, R$ denotes an Archimedean real closed field.

Concerning the direction set, let us analyse the following example:

Example 2.1. Let $R$ be the field of real algebraic numbers, and let $\left\{a_{m}\right\}$ be the sequence of points of $R^{2}$ defined by

$$
a_{m}=\left(\frac{1}{m}, \frac{1}{m}\left(1+\frac{1}{1 !}+\frac{1}{2 !}+\cdots+\frac{1}{m !}\right)\right) .
$$

Clearly $a_{m}$ tends to $0 \in R^{2}$, and $\frac{a_{m}}{\left\|a_{m}\right\|}$ tends to a pair of transcendental numbers $\left(\frac{1}{\sqrt{1+e^{2}}}, \frac{e}{\sqrt{1+e^{2}}}\right)$ which is not an element of $R^{2}$.

We can see that for any $p \in S^{1} \subset \mathbb{R}^{2}$, there is a sequence of points $\left\{a_{m}\right\}$ of $R^{2}$ tending to $0 \in R^{2}$ such that $\frac{a_{m}}{\left\|a_{m}\right\|}$ tends to $p$. Therefore we have $\operatorname{dim}_{R} R^{2}=2$, but there are infinitely many points in $S^{1} \backslash R^{2}$ which are independent over $R$.

Let $\left\{b_{m}\right\}$ be the sequence of points of $R^{2}$ defined by

$$
b_{m}=\left(0, \frac{1}{m}\left(1+\frac{1}{1 !}+\frac{1}{2 !}+\cdots+\frac{1}{m !}\right)\right) .
$$

Then we can see that there is a bi-Lipschitz homeomorphism $h:\left(R^{2}, 0\right) \rightarrow\left(R^{2}, 0\right)$ such that for all $m, h\left(a_{m}\right)=b_{m}$. On the other hand we have

$$
\lim _{m \rightarrow \infty} \frac{a_{m}}{\left\|a_{m}\right\|} \notin R^{2}, \quad \lim _{m \rightarrow \infty} \frac{b_{m}}{\left\|b_{m}\right\|} \in R^{2} .
$$

To avoid any confusion when we consider the limit, we give the precise definition of the direction set over an Archimedean real closed field $R$.

Definition 2.2. Let $A$ be a set-germ at $0 \in R^{n}$ such that $0 \in \bar{A}$. We define the direction set $D(A)$ of $A$ at $0 \in R^{n}$ by

$$
D(A):=\left\{a \in S^{n-1} \mid \exists\left\{x_{i}\right\} \subset A \backslash\{0\}, x_{i} \rightarrow 0 \in R^{n} \text { s.t. } \frac{x_{i}}{\left\|x_{i}\right\|} \rightarrow a, i \rightarrow \infty\right\} .
$$

Here $S^{n-1} \subset R^{n}$ denotes the unit sphere centred at $0 \in R^{n}$.

We denote by $L D(A)$ a half-cone of $D(A)$ with the origin $0 \in R^{n}$ as the vertex:

$$
L D(A):=\left\{t a \in R^{n} \mid a \in D(A), t \geq 0\right\} .
$$

Let us recall the definition of an o-minimal structure on a real closed field $R$. 
Definition 2.3. Let $\mathcal{D}$ be a sequence $\left(\mathcal{D}_{n}\right)_{n \in \mathbb{N}}$ where for each $n \in \mathbb{N}, \mathcal{D}_{n}$ is a family of subsets of $R^{n}$. We say that $\mathcal{D}$ is an o-minimal structure on $R$ if:

(D1) $\mathcal{D}_{n}$ is a boolean algebra.

(D2) If $A \in \mathcal{D}_{n}$, then $A \times R$ and $R \times A \in \mathcal{D}_{n+1}$.

(D3) If $A \in \mathcal{D}_{n+1}$, then $\pi(A) \in \mathcal{D}_{n}$, where $\pi: R^{n+1} \rightarrow R^{n}$ is the projection on the first $n$ coordinates.

(D4) $\mathcal{D}_{n}$ contains $\left\{x \in R^{n}: P(x)=0\right\}$ for every polynomial $P \in R\left[X_{1}, \cdots, X_{n}\right]$.

(D5) Each set in $\mathcal{D}_{1}$ is a finite union of intervals and points.

A subset $A$ of $R^{n}$ belonging to $\mathcal{D}_{n}$ is called definable in $\mathcal{D}$. A map $f: A \rightarrow R^{m}$ is definable in $\mathcal{D}$, if its graph is a definable subset of $R^{n} \times R^{m}$ in $\mathcal{D}$.

The class of semi-algebraic sets and the class of global sub-analytic sets are examples of o-minimal structures on the field of real numbers $(\mathbb{R},+, \cdot)$. We refer the readers to [4], [5] and [2] for the basic properties of o-minimal structures. In particular, the following results and properties are frequently used in our paper.

(1) The dimension of definable sets is well-defined (this follows from the Cell Decomposition Theorem ([4], Chapter $3(2.11))$ );

(2) Monotonicity ([4], Chapter 3 (1.2));

(3) Curve Selection Lemma ([4], Chapter 6 (1.5)).

We mention one more fact. The Lojasiewicz inequalities for definable sets in an ominimal structure are discussed in [5] and [10]. The Lojasiewicz inequalities in the sense of [11] hold in polynomially bounded o-minimal structures.

Before ending this section, we introduce a notation which we will often use in this paper, namely we denote by $\Phi$ the set of all odd, strictly increasing, continuous definable germs from $(R, 0)$ to $(R, 0)$. Note that, by Monotonicity, $\Phi$ is ordered by the following relation:

$$
\theta_{1} \leq \theta_{2} \text { iff } \theta_{1}(t) \leq \theta_{2}(t) \text {, for all } t>0 \text { sufficiently small. }
$$

\section{Bi-LiPschitz EQUivalenCE DOES NOT ALWAYS IMPLY DEFINABLE ONE}

In our main theorem (Theorem (1.5)) we did not assume the definability of the Lipschitz homeomorphism $h:\left(R^{n}, 0\right) \rightarrow\left(R^{n}, 0\right)$. In the case when $h$ is definable, we can easily show the theorem as follows.

Let $\bar{h}: S^{n-1} \rightarrow S^{n-1}, S^{n-1} \subset R^{n}$, be a mapping defined by

$$
\bar{h}(a)=\lim _{t \rightarrow 0} \frac{h(t a)}{\|h(t a)\|} .
$$

Then it is easy to see that $\bar{h}$ is a well-defined definable bi-Lipschitz homeomorphism. Therefore it follows that $\bar{h}(D(A))=D(h(A)))$.

Related to the above fact, it may be natural to ask if we can replace a bi-Lipschitz homeomorphism $h$ with a definable bi-Lipschitz homeomorphism $h^{\prime}$. That is to say, whether the existence of $h$ implies the existence of a definable bi-Lipschitz homeomorphism $h^{\prime}$ with 
$h^{\prime}(A)=h(A)$ and $h^{\prime}(B)=h(B)$. If the answer were positive, we would have a different proof of our main theorem, without using the main properties mentioned in the introduction. Nevertheless, the answer to this question is negative. More precisely, bi-Lipschitz equivalence does not always guarantee the existence of a definable one.

Theorem 3.1. There exist $n \in \mathbb{N}$ and compact polyhedra $A_{1}$ and $A_{2}$ in $\mathbb{R}^{n}$, such that the germs of $\left(\mathbb{R}^{n}, A_{1}\right)$ and $\left(\mathbb{R}^{n}, A_{2}\right)$ at $0 \in \mathbb{R}^{n}$ are bi-Lipschitz homeomorphic but not definably homeomorphic in any o-minimal structure on $\mathbb{R}$.

Proof. We first recall the following result of R. C. Kirby and L. C. Siebenmann [8].

For a $P L$ manifold $X_{1}$ of dimension $\geq 5$ with $H^{3}\left(X_{1} ; Z_{2}\right) \neq 0$, there exists a $P L$ manifold $X_{2}$ which is homeomorphic but not PL homeomorphic to $X_{1}$.

Let $X_{1}$ and $X_{2}$ be such compact manifolds contained in $\mathbb{R}^{m_{1}}$ and $\mathbb{R}^{m_{2}}$, respectively, and let $h: X_{1} \rightarrow X_{2}$ be a homeomorphism. On the other hand, by D. Sullivan [14, the Lipschitz manifold structure on a topological manifold of dimension $\neq 4$ is unique up to bi-Lipschitz homeomorphisms. Therefore we can choose $h$ as a bi-Lipschitz homeomorphism since a PL manifold is a Lipschitz manifold. For a point $x$ in $\mathbb{R}^{n}$ and a subset $X$ of $\mathbb{R}^{n}$, let $x * X$ denote the cone with vertex $x$ and base $X$, and let $X_{x}$ be the germ of $X$ at $x$. Set

$$
\begin{aligned}
Y_{i} & :=0 *\left(X_{i} \times\{1\}\right) \subset \mathbb{R}^{m_{i}} \times \mathbb{R} \text { for } i=1,2, \\
A_{1} & :=Y_{1} \times\{0\} \subset \mathbb{R}^{m_{1}+1} \times \mathbb{R}^{m_{2}+1}, \\
A_{2} & :=\{0\} \times Y_{2} \subset \mathbb{R}^{m_{1}+1} \times \mathbb{R}^{m_{2}+1}, \\
n & :=m_{1}+m_{2}+2 .
\end{aligned}
$$

We first show the following claim.

Claim 1. The following germs at $0,\left(\mathbb{R}^{n}, A_{1}\right)_{0}$ and $\left(\mathbb{R}^{n}, A_{2}\right)_{0}$, are bi-Lipschitz homeomorphic.

Proof. The idea of our proof comes from the proof of Proposition 10.4 in R. J. Daverman [3]. First we extend $h \times$ id $: X_{1} \times\{1\} \rightarrow X_{2} \times\{1\}$ to a bi-Lipschitz homeomorphism $h^{*}: Y_{1} \rightarrow Y_{2}$ by cone extension. To be precise, set $h^{*}(0):=0$ and

$$
h^{*}(t x, t):=(t h(x), t) \quad \text { for }(t, x) \in(0,1) \times X_{1} .
$$

Then $h^{*}$ is bijective and $\left(h^{*}\right)^{-1}(t x, t)=\left(t h^{-1}(x), t\right)$. Moreover, we can see that $h^{*}$ is Lipschitz as follows. Let $(t, x),\left(t^{\prime}, x^{\prime}\right) \in[0,1] \times X_{1}$. Then we have

$$
\begin{array}{r}
\left\|h^{*}(t x, t)-h^{*}\left(t^{\prime} x^{\prime}, t^{\prime}\right)\right\| \leq\left\|t h(x)-t^{\prime} h\left(x^{\prime}\right)\right\|+\left\|t-t^{\prime}\right\|, \\
\left\|t h(x)-t^{\prime} h\left(x^{\prime}\right)\right\| \leq\left\|t h(x)-t^{\prime} h(x)\right\|+\left\|t^{\prime} h(x)-t^{\prime} h\left(x^{\prime}\right)\right\| \\
\leq c\left\|t-t^{\prime}\right\|+c t^{\prime}\left\|x-x^{\prime}\right\|, \\
t^{\prime}\left\|x-x^{\prime}\right\| \leq\left\|t x-t^{\prime} x\right\|+\left\|t x-t^{\prime} x^{\prime}\right\| \leq c\left\|t-t^{\prime}\right\|+\left\|t x-t^{\prime} x^{\prime}\right\|
\end{array}
$$

for some constant real number $c>0$. Hence we have

$$
\left\|h^{*}(t x, t)-h^{*}\left(t^{\prime} x^{\prime}, t^{\prime}\right)\right\| \leq c^{\prime}\left\|t-t^{\prime}\right\|+c^{\prime}\left\|t x-t^{\prime} x^{\prime}\right\|
$$


for some constant real number $c^{\prime}>0$. In the same way we can see that $\left(h^{*}\right)^{-1}$ is Lipschitz. Thus $h^{*}$ is bi-Lipschitz.

Secondly we extend $h^{*}$ to a Lipschitz (not bi-Lipschitz) map $\tilde{h}: \mathbb{R}^{m_{1}+1} \rightarrow \mathbb{R}^{m_{2}+1}$. Let $K$ be a simplicial decomposition of $\mathbb{R}^{m_{1}+1}$ such that $Y_{1}$ is the underlying polyhedron of a full subcomplex $K_{1}$ of $K . K_{1}$ is called full in $K$ if each simplex in $K$ with all its vertices in $K_{1}$ is necessarily contained in $K_{1}$. (When $K_{1}$ is not full in $K$, we replace $K$ and $K_{1}$ with their barycentric subdivisions $K^{\prime}$ and $K_{1}^{\prime}$. Then $K_{1}^{\prime}$ is full in $K^{\prime}$. See C. P. Rourke and B. J. Sanderson [12] for details.) Let $K^{r}$ denote the $r$-skeleton of $K$, namely, the simplexes in $K$ of dimension $\leq r$. We define $\tilde{h}$ on the underlying polyhedron $\left|K^{r}\right|$ of $K^{r}$ by induction on $r$. If $r=0$, set $\tilde{h}:=0$ on $\left|K^{0}\right|-Y_{1}$. Assume that $\tilde{h}$ is already defined on $\left|K^{r-1}\right|$ for some $r>0$. For each $\sigma \in K^{r}-K^{r-1}$ with $\sigma \not \subset Y_{1}$, let $v_{0}, \cdots, v_{r}$ be the vertices of $\sigma$ such that $v_{0} \notin Y_{1}$, which exists by the fullness of $K_{1}$. Note that $v_{0}, v_{1} * \cdots * v_{r} \in K^{r-1}$. Set

$$
\begin{array}{r}
\tilde{h}\left(\sum_{i=0}^{r} t_{i} v_{i}\right):=\sum_{k=1}^{r} t_{k} \tilde{h}\left(\sum_{i=1}^{r} t_{i} v_{i} / \sum_{j=1}^{r} t_{j}\right) \\
\text { for }\left(t_{0}, \ldots, t_{r}\right) \in[0,1]^{r+1} \text { with } \sum_{i=0}^{r} t_{i}=1 \text { and } \sum_{i=1}^{r} t_{i} \neq 0,
\end{array}
$$

which is well-defined because $\sum_{i=1}^{r} t_{i} v_{i} / \sum_{j=1}^{r} t_{j} \in v_{1} * \cdots * v_{r}$. Then $\tilde{h}$ is a map from $\left|K^{r}\right|$ to $\mathbb{R}^{m_{2}+1}$ and we claim that it is Lipschitz. In order to see this, it suffices to show that $\left.\tilde{h}\right|_{\sigma}$ is Lipschitz for the above $\sigma$, because $\tilde{h}=0$ outside of a compact neighbourhood of $Y_{1}$ in $\mathbb{R}^{m_{1}+1}$. By the above definition of $\left.\tilde{h}\right|_{\sigma},\left.\tilde{h}\right|_{\sigma}\left(v_{0}\right)=0$ and $\left.\tilde{h}\right|_{\sigma}$ is the cone extension of $\left.\tilde{h}\right|_{v_{1} * \cdots * v_{r}}$. Hence, as shown above, $\left.\tilde{h}\right|_{\sigma}$ is Lipschitz since so is $\left.\tilde{h}\right|_{v_{1} * \cdots * v_{r}}$.

Set $A_{3}:=$ graph $h^{*} \subset \mathbb{R}^{m_{1}+1} \times \mathbb{R}^{m_{2}+1}$. We shall prove that $\left(\mathbb{R}^{n}, A_{3}\right)_{0}$ is bi-Lipschitz homeomorphic to $\left(\mathbb{R}^{n}, A_{1}\right)_{0}$ and $\left(\mathbb{R}^{n}, A_{2}\right)_{0}$. Consider $\left(\mathbb{R}^{n}, A_{3}\right)_{0}$ and $\left(\mathbb{R}^{n}, A_{1}\right)_{0}$. Set

$$
\phi(x, y):=(x, y-\tilde{h}(x)) \quad \text { for }(x, y) \in \mathbb{R}^{m_{1}+1} \times \mathbb{R}^{m_{2}+1} .
$$

Then $\phi$ is a homeomorphism of $\mathbb{R}^{n}, \phi^{-1}\left(A_{1}\right)=A_{3}, \phi(0)=0$, and $\phi$ is Lipschitz since so is $\tilde{h}$. In addition, $\phi$ has its inverse given by

$$
\mathbb{R}^{m_{1}+1} \times \mathbb{R}^{m_{2}+1} \ni(x, y) \rightarrow(x, y+\tilde{h}(x)) \in \mathbb{R}^{m_{1}+1} \times \mathbb{R}^{m_{2}+1},
$$

which is also Lipschitz. Thus $\phi$ is a bi-Lipschitz homeomorphism from $\left(\mathbb{R}^{n}, A_{3}\right)_{0}$ to $\left(\mathbb{R}^{n}, A_{1}\right)_{0}$. In the same way, by extending $\left(h^{*}\right)^{-1}$ to a Lipschitz map from $\mathbb{R}^{m_{2}+1}$ to $\mathbb{R}^{m_{1}+1}$, we see that $\left(\mathbb{R}^{n}, A_{3}\right)_{0}$ and $\left(\mathbb{R}^{n}, A_{2}\right)_{0}$ are bi-Lipschitz homeomorphic.

We next show the following claim.

Claim 2. $\left(\mathbb{R}^{n}, A_{1}\right)_{0}$ and $\left(\mathbb{R}^{n}, A_{2}\right)_{0}$ are not definably homeomorphic.

Proof. Assume that they are definably homeomorphic. Then $\left(A_{1}\right)_{0}$ and $\left(A_{2}\right)_{0}$ are definably homeomorphic. Hence shrinking $A_{1}$ we have a definable embedding $f: A_{1} \rightarrow A_{2}$ such that $f(0)=0$ and $f\left(A_{1}\right)$ is a neighbourhood of 0 in $A_{2}$.

Here we recall the following facts from [13]. 
Definable Triangulation Theorem. (Theorem II.2.1 in [13]) Any compact definable set $X$ is definably homeomorphic to some polyhedron $X^{\prime}$.

Remark 3.2. (Remark II.2.3 in [13]) If the above $X$ is contained in the underlying polyhedron of a finite simplicial complex $K$, then we can choose $X^{\prime}$ in $|K|$ and a definable homeomorphism $g: X \rightarrow X^{\prime}$ so that $g(X \cap|\sigma|) \subset|\sigma|$ for each $\sigma \in K$.

Definable Hauptvermutung. (Corollary III.1.4 in [13]) Any two compact polyhedra are $P L$ homeomorphic if they are definably homeomorphic.

Applying the definable triangulation theorem and Remark 3.2 to $f\left(A_{1}\right)$ and 0 , we can assume that $f\left(A_{1}\right)$ is a polyhedron. Therefore we may assume $f\left(A_{1}\right)=A_{2}$ from the beginning. The links of $A_{1}$ and $A_{2}$ at 0 are $X_{1}$ and $X_{2}$ respectively. By our choice of $X_{1}$, they are not PL homeomorphic to a sphere or to a ball, hence $A_{1}$ and $A_{2}$ are not $\mathrm{PL}$ manifolds at 0 (0 is a singular point). On the other hand the links at the other points are all PL homeomorphic to a sphere or to a ball. By the definable Hauptvermutung, $A_{1}$ and $A_{2}$ are $\mathrm{PL}$ homeomorphic as polyhedra. Moreover, because the origin 0 is the only singular point of $A_{1}$ and $A_{2}$, the PL homeomorphism has to carry 0 to 0 . Thus $\left(A_{1}\right)_{0}$ and $\left(A_{2}\right)_{0}$ are $\mathrm{PL}$ homeomorphic, which is a contradiction because of our our choice of $X_{1}$ and $X_{2}$.

This completes the proof of the theorem.

Remark 3.3. In the proof of Claim 1 we constructed a Lipschitz extension of $h^{*}$ to $\tilde{h}$ using the cone structure. However, in general, to extend a Lipschitz map is not difficult. Indeed, for a Lipschitz function with constant $L, f: A \rightarrow \mathbb{R}, A \subset X, A$ endowed with the induced metric from $(X, d)$, we have an extension formula (see S. Banach [1]):

$$
\alpha(x):=\inf _{a \in A}(f(a)+L d(x, a)) .
$$

Similarly one can extend it by

$$
\beta(x):=\sup _{a \in A}(f(a)-L d(x, a)) .
$$

Note that $\beta(x) \leq \alpha(x)$. Any convex combination $t \alpha(x)+(1-t) \beta(x), 0 \leq t \leq 1$, also gives a Lipschitz extension.

This construction can be used to extend Lipschitz maps as well, however, without preserving the Lipschitz constant.

\section{Sea-Tangle Properties in o-minimal Structures}

We recall the notion of sea-tangle neighbourhood for a subset of $\mathbb{R}^{n}$, originated from the classical notion of horn-neighbourhood for an analytic set or more generally a subanalytic set in $\mathbb{R}^{n}$. 
Definition 4.1. Let $A \subset \mathbb{R}^{n}$ such that $0 \in \bar{A}$, and let $d, C>0$. The sea-tangle neighbourhood $S T_{d}(A ; C)$ of $A$, of degree $d$ and width $C$, is defined by:

$$
S T_{d}(A ; C):=\left\{x \in \mathbb{R}^{n} \mid \operatorname{dist}(x, A) \leq C\|x\|^{d}\right\} .
$$

See $\S 4$ of [9] for some sea-tangle properties. For instance, the following is shown.

Proposition 4.2. ([9] Proposition 4.7) Let $A$ be a subanalytic set-germ at $0 \in \mathbb{R}^{n}$ such that $0 \in \bar{A}$. Then there is $d_{1}>1$ such that $A \subset S T_{d}(L D(A) ; C)$ as set-germs at $0 \in \mathbb{R}^{n}$ for any $d$ with $1<d<d_{1}$ and $C>0$.

Both Hironaka's selection lemma for subanalytic sets and a Lojasiewicz inequality have played an important role in the proof of the above result. As mentioned in $\S 2$, it is known that the curve selection lemma holds also for definable sets in an o-minimal structure; on the other hand, the usual Lojasiewicz inequality does not always hold in an o-minimal structure. Accordingly, Proposition 4.2 might be false for a definable set in some ominimal structure. Indeed, the following example confirms this.

Example 4.3. Let $\pi: \mathcal{M}_{2} \rightarrow \mathbb{R}^{2}$ be a blowing-up at $(0,0) \in \mathbb{R}^{2}$, and let $a=(0,1) \in S^{1}$. We denote by $L(a)$ the half line in $\mathbb{R}^{2}$ with the origin as the starting point passing through $a$ and by $\hat{L}(a)$ the strict transform of $L(a)$ in $\mathcal{M}_{2}$ by $\pi$. In a suitable coordinate neighbourhood, $\pi: \mathbb{R}_{(X, Y)}^{2} \rightarrow \mathbb{R}^{2}$ can be expressed as $\pi(X, Y)=(X Y, Y)$. Here $(0,0) \in$ $\mathbb{R}_{(X, Y)}^{2}$ is the intersection of $\hat{L}(a)$ and the exceptional divisor $E=\pi^{-1}(0,0)$.

Let $B:=\left\{(X, Y) \in \mathbb{R}_{(X, Y)}^{2} \mid Y=e^{-\frac{1}{|X|^{2}}}, X \geq 0\right\}$. Then the curve $B$ is not contained in $\left\{\left.(X, Y) \in \mathbb{R}_{(X, Y)}^{2}|| Y\left|\geq C^{\prime}\right| X\right|^{d^{\prime}}\right\}$ as germs at $(0,0) \in \mathbb{R}_{(X, Y)}^{2}$, for any $d^{\prime}>0, C^{\prime}>0$.

Let $\mathbb{R}_{\text {exp }}$ be Wilkie's exponential field ([15]), and let $\mathcal{D}$ be the o-minimal structure on it. Set $A:=\pi(B)$. Then we can see that $A \in \mathcal{D}$ and $L D(A)=L(a)$, but $A$ is not contained in any sea-tangle neighbourhood $S T_{d}(L(a) ; C)$ as germs at $(0,0) \in \mathbb{R}^{2}$, for $d>1, C>0$. Therefore Proposition 4.2 does not hold for this definable set $A$ in the o-minimal structure $\mathcal{D}$.

Taking into account the above fact, in order to develop sea-tangle properties in an o-minimal structure on an Archimedean real closed field $R$, the definition of sea-tangle neighbourhood has to be modified. Note that $R^{n}$ has an induced metric from $\mathbb{R}^{n}$. ¿From now on let us fix an o-minimal structure on $R$. Here we recall that $\Phi$ is the set of all odd, strictly increasing, continuous definable germs from $(R, 0)$ to $(R, 0)$. Then we define the notion of sea-tangle neighbourhood of a definable set as follows:

Definition 4.4. Let $A \subset R^{n}$ such that $0 \in \bar{A}$, and let $\theta \in \Phi$. The sea-tangle neighbourhood $S T_{\theta}(A)$ of $A$ with respect to $\theta$ is defined by:

$$
S T_{\theta}(A):=\left\{x \in R^{n} \mid \operatorname{dist}(x, A) \leq \theta(\|x\|)\|x\|\right\} .
$$

Remark 4.5. (1) Let $x \in R^{n}$ and $A \subset R^{n}$. In general, dist $(x, A)=\inf _{a \in A} d(x, a)$ does not always belong to $R$; nonetheless it is always a non-negative real number.

(2) If $A$ is definable, then $D(A), L D(A)$ and $S T_{\theta}(A)$ are also definable.

Let $\mathcal{S}$ be the set of set-germs $A \subset R^{n}$ at $0 \in R^{n}$ such that $0 \in \bar{A}$. 
Definition 4.6. Let $A, B \in \mathcal{S}$. We say that $A$ and $B$ are $S T$-equivalent, if there are $\theta_{1}, \theta_{2} \in \Phi$ such that $B \subset S T_{\theta_{1}}(A)$ and $A \subset S T_{\theta_{2}}(B)$ as germs at $0 \in R^{n}$. We write $A \underset{\mathrm{st}}{\sim} B$. Remark 4.7. $S T$-equivalence $\underset{\mathrm{st}}{\sim}$ is an equivalence relation in $\mathcal{S}$.

We first describe several sea-tangle properties for general subsets of $R^{n}$.

Let $\phi:\left(R^{n}, 0\right) \rightarrow\left(R^{n}, 0\right)$ be a bi-Lipschitz homeomorphism, namely there are positive numbers $K_{1}, K_{2} \in R$ with $0<K_{1} \leq K_{2}$ such that

$$
K_{1}\left\|x_{1}-x_{2}\right\| \leq\left\|\phi\left(x_{1}\right)-\phi\left(x_{2}\right)\right\| \leq K_{2}\left\|x_{1}-x_{2}\right\|
$$

in a small neighbourhood of $0 \in R^{n}$. Conversely, we have

$$
\frac{1}{K_{2}}\left\|y_{1}-y_{2}\right\| \leq\left\|\phi^{-1}\left(y_{1}\right)-\phi^{-1}\left(y_{2}\right)\right\| \leq \frac{1}{K_{1}}\left\|y_{1}-y_{2}\right\|
$$

in a small neighbourhood of $0 \in R^{n}$. With these Lipschitz constants we can formulate the following Sandwich Lemma.

Lemma 4.8. Let $A \subset R^{n}$ such that $0 \in \bar{A}$. Then, for $\theta \in \Phi$,

(i) $\phi\left(S T_{\theta}(A)\right) \subset S T_{\theta_{1}}(\phi(A))$ where $\theta_{1}(t)=\frac{K_{2}}{K_{1}} \theta\left(\frac{t}{K_{1}}\right) \in \Phi$. and

(ii) $S T_{\theta_{2}}(\phi(A)) \subset \phi\left(S T_{\theta}(A)\right)$ where $\theta_{2}(t)=\frac{K_{1}}{K_{2}} \theta\left(\frac{t}{K_{2}}\right) \in \Phi$

in a small neighbourhood of $0 \in R^{n}$.

The next proposition follows from the above Sandwich Lemma.

Proposition 4.9. ST-equivalence is preserved by a bi-Lipschitz homeomorphism.

In [9] there are mentioned some directional properties for the original notion of seatangle neighbourhood $S T_{d}(A ; C)$. The same properties hold also for our sea-tangle neighbourhood $S T_{\theta}(A)$. Throughout this section, let $A, B \subset R^{n}$ be set-germs at $0 \in R^{n}$ such that $0 \in \bar{A} \cap \bar{B}$, namely $A, B \in \mathcal{S}$, and let $h:\left(R^{n}, 0\right) \rightarrow\left(R^{n}, 0\right)$ be a bi-Lipschitz homeomorphism.

Lemma 4.10. Suppose that there is $\theta \in \Phi$ such that $A \subset S T_{\theta}(B)$ as set-germs at $0 \in R^{n}$. Then we have $D(h(A)) \subset D(h(B))$. In addition, we have $D\left(S T_{\theta^{\prime}}(h(A))\right) \subset D(h(B))$ for any $\theta^{\prime} \in \Phi$.

Proof. Let $a \in D(h(A))$. Then there is a sequence of points $\left\{a_{m}\right\} \subset A$ tending to $0 \in R^{n}$ such that $\lim _{m \rightarrow \infty} \frac{h\left(a_{m}\right)}{\left\|h\left(a_{m}\right)\right\|}=a$. By assumption, $A \subset S T_{\theta}(B)$. Therefore, for each $m$ we can take $b_{m} \in B$ such that

$$
\left\|a_{m}-b_{m}\right\| \ll\left\|a_{m}\right\|,\left\|b_{m}\right\| .
$$

Since $h$ is a bi-Lipschitz homeomorphism,

$$
\left\|h\left(a_{m}\right)-h\left(b_{m}\right)\right\| \ll\left\|h\left(a_{m}\right)\right\|,\left\|h\left(b_{m}\right)\right\| .
$$

Thus we have

$$
a=\lim _{m \rightarrow \infty} \frac{h\left(a_{m}\right)}{\left\|h\left(a_{m}\right)\right\|}=\lim _{m \rightarrow \infty} \frac{h\left(b_{m}\right)}{\left\|h\left(b_{m}\right)\right\|} \in D(h(B)) .
$$

The second statement follows from the first one. 
We have the following corollary.

Corollary 4.11. (1) $D\left(S T_{\theta}(A)\right)=D(A)$ for any $\theta \in \Phi$.

(2) $D\left(S T_{\theta}(h(A))\right)=D(h(A))$ for any $\theta \in \Phi$.

(3) If $A$ and $B$ are ST-equivalent, then we have $D(A)=D(B)$ and $D(h(A))=$ $D(h(B))$.

For a definable set we have more specific sea-tangle properties. Proposition 4.2 is modified to the following.

Proposition 4.12. Let $A$ be a definable set-germ at $0 \in R^{n}$ such that $0 \in \bar{A}$. Then there is $\theta \in \Phi$ such that $A \subset S T_{\theta}(L D(A))$ as set-germs at $0 \in R^{n}$.

Proof. Let $g(x)=\frac{d(x, L D(A))}{\|x\|}$ for $x \in \bar{A}, x \neq 0$.

Claim. $\lim _{x \rightarrow 0} g(x)=0$.

Proof. If the claim does not hold, then by the Curve selection lemma, there exist $c>0$ and a definable curve $\gamma:(0,1) \rightarrow \bar{A} \backslash\{0\}$ such that $\lim _{t \rightarrow 0} \gamma(t)=0$ and $\frac{d(\gamma(t), L D(A))}{\|\gamma(t)\|} \geq c$ for sufficiently small $t>0$. By Monotonicity, $\lim _{t \rightarrow 0} \frac{\gamma(t)}{\|\gamma(t)\|}=a \in D(A)$. Therefore we have

$$
\frac{d(\gamma(t), L D(A))}{\|\gamma(t)\|} \leq \frac{d(\gamma(t), L(a))}{\|\gamma(t)\|} \rightarrow 0
$$

when $t \rightarrow 0$. This is a contradiction.

By this claim, $g$ can be naturally extended to a continuous definable function $g: \bar{A} \rightarrow R$ with $g(0)=0$. By the Lojasiewicz inequality $([5])$, there exists $\theta \in \Phi$ such that $g(x) \leq$ $\theta(\|x\|)$ for $x \in A$ near $0 \in R^{n}$. This means that $A \subset S T_{\theta}(L D(A))$ as germs at $0 \in R^{n}$.

Proposition 4.13. Suppose that $A$ is definable. Then, for any $\theta_{1} \in \Phi$, there is $\theta_{2} \in \Phi$ such that $S T_{\theta_{1}}(L D(A)) \subset S T_{\theta}(A)$ as germs at $0 \in R^{n}$, for any $\theta \in \Phi$ with $\theta \geq \theta_{2}$.

Proof. Let $\theta_{1} \in \Phi$. Then, by Corollary 4.11 (1), we have $D\left(S T_{\theta_{1}}(L D(A))=D(L D(A))=\right.$ $D(A)$. Using the same arguments for $g(x)=\frac{d(x, A)}{\|x\|}\left(x \in S T_{\theta_{1}}(L D(A)), x \neq 0\right)$ as in the proof of Proposition 4.12, we can find $\theta_{2} \in \Phi$ such that $S T_{\theta_{1}}(L D(A)) \subset S T_{\theta_{2}}(A)$. Hence $L D(A) \subset S T_{\theta_{2}}(A)$ as germs at $0 \in R^{n}$.

By Propositions 4.12, 4.13, we have:

Theorem 4.14. If $A$ is definable, then $A$ is $S T$-equivalent to $L D(A)$.

In a similar way to Corollary 4.15 in [9], using Propositions 4.12, 4.13 and Lemma 4.8, we can show the following result:

Corollary 4.15. Suppose that $h(A), h(B)$ are definable. If $D(h(A)) \subset D(h(B))$, then there is $\theta \in \Phi$ such that $A \subset S T_{\theta}(B)$ as germs at $0 \in R^{n}$.

By Corollary 4.15 and Lemma 4.10, we have:

Theorem 4.16. Suppose that $h(A), h(B)$ are definable. Then the following conditions are equivalent.

(1) $D(h(A)) \subset D(h(B))$.

(2) There is $\theta \in \Phi$ such that $A \subset S T_{\theta}(B)$ as germs at $0 \in R^{n}$. 


\section{Sequence Selection Property}

In this section we discuss directional properties of sets with the sequence selection property, denoted by (SSP) for short, over an Archimedean real closed field $R$. The set $h(L D(A))$ takes a very important role in the proof of our main theorem. In [9] it is shown that over the field of real numbers $\mathbb{R}, h(L D(A))$ satisfies condition (SSP) provided that $A$ and $h(A)$ are both subanalytic. We give an improvement of this result here.

Let us recall the notion of sequence selection property.

Definition 5.1. Let $A \subset R^{n}$ be a set-germ at $0 \in R^{n}$ such that $0 \in \bar{A}$. We say that $A$ satisfies condition $(S S P)$, if for any sequence of points $\left\{a_{m}\right\}$ of $R^{n}$ tending to $0 \in R^{n}$ such that $\lim _{m \rightarrow \infty} \frac{a_{m}}{\left\|a_{m}\right\|} \in D(A)$, there is a sequence of points $\left\{b_{m}\right\} \subset A$ such that

$$
\left\|a_{m}-b_{m}\right\| \ll\left\|a_{m}\right\|,\left\|b_{m}\right\| .
$$

We have some remarks on $(S S P)$ (cf. [9]).

Remark 5.2. Condition $(S S P)$ is $C^{1}$ invariant, but not bi-Lipschitz invariant.

Remark 5.3. Let $A \subset R^{n}$ be a set-germ at $0 \in R^{n}$ such that $0 \in \bar{A}$.

(1) The cone $L D(A)$ satisfies condition $(S S P)$.

(2) If $A \subset \mathbb{R}^{n}$ is subanalytic, then it satisfies condition $(S S P)$.

(3) If $A \subset R^{n}$ is definable in an o-minimal structure, then it satisfies condition (SSP).

Remark 5.4. We can describe condition $(S S P)$ without using the convergence of a sequence of points as follows:

$$
\forall \epsilon>0 \in R \forall \delta>0 \in R \forall x \neq 0\left(\|x\| \leq \delta, \operatorname{dist}\left(\frac{x}{\|x\|}, D(A)\right) \leq \epsilon, \exists y \in A,\|x-y\| \leq \epsilon\|x\|\right)
$$

We recall the following lemma.

Lemma 5.5. (Lemma 5.6 in $[9])$ Let $h:\left(\mathbb{R}^{n}, 0\right) \rightarrow\left(\mathbb{R}^{n}, 0\right)$ be a bi-Lipschitz homeomorphism, and let $A \subset \mathbb{R}^{n}$ such that $0 \in \bar{A}$. Then $D(h(A)) \subset D(h(L D(A)))$. If A satisfies condition (SSP), the equality holds.

In order to show the above lemma, the following property was used in [9] :

Let $\left\{a_{m}\right\}$ be a sequence of points of $\mathbb{R}^{n}$ tending to $0 \in \mathbb{R}^{n}$. Then there is a subsequence of points $\left\{a_{k}\right\}$ of $\left\{a_{m}\right\}$ such that

$$
\lim _{k \rightarrow \infty} \frac{a_{k}}{\left\|a_{k}\right\|} \in S^{n-1}=\left\{x \in \mathbb{R}^{n} \mid\|x\|=1\right\} .
$$

This property does not always hold on an Archimedean real closed field $R$. In fact, Lemma 5.5 is false for the real closed field of algebraic numbers. Let us recall Example 2.1. Let $A=\left\{a_{m}\right\}$. Then $h(A)=\left\{b_{m}\right\}$. Since $D(A)=\emptyset$ we have $D(h(L D(A)))=\emptyset$. Nevertheless $D(h(A))=\{(0,1)\}$.

Over an Archimedean real closed field, we can show the following weaker result, which is enough to show our main theorem.

Lemma 5.6. Let $h:\left(R^{n}, 0\right) \rightarrow\left(R^{n}, 0\right)$ be a bi-Lipschitz homeomorphism, and let $A \subset R^{n}$ such that $0 \in \bar{A}$. Suppose that $A$ is definable. Then we have $L D(h(A))=L D(h(L D(A)))$. 
Proof. We first show the inclusion $L D(h(A)) \subset L D(h(L D(A)))$. By Proposition 4.12, there is $\theta_{1} \in \Phi$ such that $A \subset S T_{\theta_{1}}(L D(A))$ as set-germs at $0 \in R^{n}$. Then, by the sandwich lemma, we have

$$
h(A) \subset h\left(S T_{\theta_{1}}(L D(A))\right) \subset S T_{\theta_{2}}(h(L D(A)))
$$

for some $\theta_{2} \in \Phi$. Therefore it follows from Corollary 4.11 (2) that

$$
L D(h(A)) \subset L D\left(S T_{\theta_{2}}(h(L D(A)))\right)=L D(h(L D(A))) .
$$

The opposite inclusion $L D(h(A)) \supset L D(h(L D(A)))$ follows from a similar argument, replacing Proposition 4.13 with Proposition 4.12 .

As a corollary of Lemma 5.6 and Remark 4.5 we have the following lemma.

Lemma 5.7. Let $h:\left(R^{n}, 0\right) \rightarrow\left(R^{n}, 0\right)$ be a bi-Lipschitz homeomorphism, and let $A \subset R^{n}$ such that $0 \in \bar{A}$. If $A$ and $h(A)$ are definable, then $L D(h(L D(A)))=L D(h(A))$ is definable.

We give one more example having condition $(S S P)$. Using a similar argument to Proposition 6.4 in [9], we can show the following:

Proposition 5.8. Let $h:\left(R^{n}, 0\right) \rightarrow\left(R^{n}, 0\right)$ be a bi-Lipschitz homeomorphism, and let $A$, $h(A) \subset R^{n}$ be definable set-germs at $0 \in R^{n}$ such that $0 \in \bar{A}$. Then the set $h(L D(A))$ satisfies condition $(S S P)$.

Let us discuss more on the sequence selection property over the field of real numbers $\mathbb{R}$. In this note we consider also the notion of weak sequence selection property, denoted by $(W S S P)$ for short.

Definition 5.9. Let $A \subset \mathbb{R}^{n}$ be a set-germ at $0 \in \mathbb{R}^{n}$ such that $0 \in \bar{A}$. We say that $A$ satisfies condition $(W S S P)$, if for any sequence of points $\left\{a_{m}\right\}$ of $\mathbb{R}^{n}$ tending to $0 \in \mathbb{R}^{n}$ such that $\lim _{m \rightarrow \infty} \frac{a_{m}}{\left\|a_{m}\right\|} \in D(A)$, there exist a subsequence $\left\{m_{j}\right\}$ and $\left\{b_{m_{j}}\right\} \subset A$ such that

$$
\left\|a_{m_{j}}-b_{m_{j}}\right\| \ll\left\|a_{m_{j}}\right\|,\left\|b_{m_{j}}\right\| .
$$

We have the following characterisation of condition $(S S P)$.

Lemma 5.10. Let $A \subset \mathbb{R}^{n}$ be a set-germ at $0 \in \mathbb{R}^{n}$ such that $0 \in \bar{A}$. If $A$ satisfies condition $(W S S P)$, then it satisfies condition $(S S P)$. Namely, conditions $(S S P)$ and $(W S S P)$ are equivalent.

Proof. We show that $(W S S P)$ implies $(S S P)$. Assume that $A$ does not satisfy condition $(S S P)$. Then there is a sequence of points $\left\{a_{m}\right\}$ tending to $0 \in \mathbb{R}^{n}$ with $\lim _{m \rightarrow \infty} \frac{a_{m}}{\left\|a_{m}\right\|} \in$ $D(A)$ such that for any sequence of points $\left\{b_{m}\right\} \subset A$, the following is not satisfied:

$$
\left\|a_{m}-b_{m}\right\| \ll\left\|a_{m}\right\| \text {. }
$$

Therefore there is a subsequence of points $\left\{a_{m_{j}}\right\}$ of $\left\{a_{m}\right\}$ such that $\lim _{m_{j} \rightarrow \infty} \frac{d\left(a_{m_{j}}, A\right)}{\left\|a_{m_{j}}\right\|}=$ $\alpha>0$, where $d\left(a_{m_{j}}, A\right)$ denotes the distance between $a_{m_{j}}$ and $A$. Taking this $\left\{a_{m_{j}}\right\}$ as the first $\left\{a_{m}\right\}$, we can assume from the beginning that $\lim _{m \rightarrow \infty} \frac{d\left(a_{m}, A\right)}{\left\|a_{m}\right\|}=\alpha>0$. This implies that there does not exist a sequence of points $\left\{b_{m_{j}}\right\} \subset A$ such that $\left\|a_{m_{j}}-b_{m_{j}}\right\| \ll\left\|a_{m_{j}}\right\|$. Therefore $A$ does not satisfy condition $(W S S P)$. 
Using Lemmas 5.5 and 5.10 we can improve Proposition 6.4 in [9] as follows:

Theorem 5.11. Let $h:\left(\mathbb{R}^{n}, 0\right) \rightarrow\left(\mathbb{R}^{n}, 0\right)$ be a bi-Lipschitz homeomorphism, and let $A \subset \mathbb{R}^{n}$ such that $0 \in \bar{A}$. Assume that $A$ satisfies condition $(S S P)$. Then $h(A)$ satisfies condition $(S S P)$, if and only if, $h(L D(A))$ satisfies condition $(S S P)$.

Proof. We first show the "only if" part. By assumption, $A$ satisfies condition $(S S P)$. Therefore it follows from Lemma 5.5 that $D(h(L D(A)))=D(h(A))$. Let $\left\{y_{m}\right\}$ be an arbitrary sequence of points of $\mathbb{R}^{n}$ tending to $0 \in \mathbb{R}^{n}$ such that

$$
\lim _{m \rightarrow \infty} \frac{y_{m}}{\left\|y_{m}\right\|} \in D(h(L D(A)))=D(h(A)) .
$$

Let $y_{m}=h\left(x_{m}\right)$ for each $m$. Since $h(A)$ satisfies condition $(S S P)$, there is a sequence of points $\left\{z_{m}\right\} \subset A$ such that

$$
\left\|h\left(x_{m}\right)-h\left(z_{m}\right)\right\| \ll\left\|h\left(x_{m}\right)\right\|,\left\|h\left(z_{m}\right)\right\| .
$$

On the other hand, there is a subsequence $\left\{z_{m_{j}}\right\}$ of $\left\{z_{m}\right\}$ such that $\lim _{m_{j} \rightarrow \infty} \frac{z_{m_{j}}}{\left\|z_{m_{j}}\right\|} \in$ $D(A)$. Since $L D(A)$ satisfies condition $(S S P)$, there is a sequence of points $\left\{\theta_{m_{j}}\right\} \subset$ $L D(A)$ such that

$$
\left\|z_{m_{j}}-\theta_{m_{j}}\right\| \ll\left\|z_{m_{j}}\right\|,\left\|\theta_{m_{j}}\right\| .
$$

It follows from the bi-Lipschitz of $h$ that

$$
\left\|h\left(z_{m_{j}}\right)-h\left(\theta_{m_{j}}\right)\right\| \ll\left\|h\left(z_{m_{j}}\right)\right\|,\left\|h\left(\theta_{m_{j}}\right)\right\| .
$$

Then we have

$$
\left\|h\left(x_{m_{j}}\right)-h\left(\theta_{m_{j}}\right)\right\| \leq\left\|h\left(x_{m_{j}}\right)-h\left(z_{m_{j}}\right)\right\|+\left\|h\left(z_{m_{j}}\right)-h\left(\theta_{m_{j}}\right)\right\| \ll\left\|h\left(z_{m_{j}}\right)\right\| .
$$

Therefore we have

$$
\left\|h\left(x_{m_{j}}\right)-h\left(\theta_{m_{j}}\right)\right\| \ll\left\|h\left(x_{m_{j}}\right)\right\|,\left\|h\left(\theta_{m_{j}}\right)\right\| .
$$

Thus $h(L D(A))$ satisfies condition (WSSP), and also condition $(S S P)$ by Lemma 5.10 .

The "if" part can be proved in a similar way.

\section{Proof of Main Theorem}

Our main theorem is proved in the same way as Theorem 1.4. Since the reduction arguments in $\S 6$ of [9] work also for definable sets, it only suffices to show the following proposition.

Proposition 6.1. Let $h:\left(R^{n}, 0\right) \rightarrow\left(R^{n}, 0\right)$ be a bi-Lipschitz homeomorphism, and let $A \subset R^{n}$ such that $0 \in \bar{A}$. Suppose that $A, h(A)$ are definable. Then

$$
\operatorname{dim} h(L D(A)) \geq \operatorname{dim} L D(h(L D(A))) .
$$


As mentioned in the introduction, Theorem 1.4 was proved using sea-tangle properties, sequence selection properties and volume arguments. In this section we discuss volume arguments and give a proof of our main theorem. In order to avoid considering the volume on a general Archimedean real closed field $R$, we take the closure of a subset of $R^{n}$ in $\mathbb{R}^{n}$. Note that $R^{n}$ is dense in $\mathbb{R}^{n}$.

For a subset $A$ of $R^{n}\left(\subset \mathbb{R}^{n}\right)$, let $\bar{A}^{\mathbb{R}}$ denote the closure of $A$ in $\mathbb{R}^{n}$ (not in $R^{n}$ ), and let $B_{\epsilon}(0)$ denote a closed $\epsilon$ ball in $\mathbb{R}^{n}$ centred at $0 \in \mathbb{R}^{n}$ for $\epsilon>0, \epsilon \in \mathbb{R}$.

Let $f, g:[0, \delta) \rightarrow \mathbb{R}, \delta>0$, be non-negative functions, where $[0, \delta)$ is a half open interval of $\mathbb{R}$. If there are real numbers $K>0,0<\delta_{1} \leq \delta$ such that

$$
f(\epsilon) \leq K g(\epsilon) \text { for } 0 \leq \epsilon \leq \delta_{1},
$$

then we write $f \precsim g$ (or $g \succsim f$ ). If $f \precsim g$ and $f \succsim g$, we write $f \approx g$.

We can easily see the following property on volumes.

Lemma 6.2. Let $A \subset R^{n}$ such that $0 \in \bar{A}$. Then

$$
\operatorname{Vol}\left(\overline{S T_{c \theta}(A)}{ }^{\mathbb{R}} \cap B_{\varepsilon}(0)\right) \approx \operatorname{Vol}\left(\overline{S T_{\theta}(A)}{ }^{\mathbb{R}} \cap B_{\varepsilon}(0)\right)
$$

for $\theta \in \Phi$ and $c>0$.

Using a similar argument as in Lemma 7.1 of [9], we can show the following lemma.

Lemma 6.3. Let $\alpha, \beta$ be linear subspaces of $R^{n}$. Suppose that $\operatorname{dim} \alpha<\operatorname{dim} \beta$. Then, for $\theta \in \Phi$,

$$
\lim _{\epsilon \rightarrow 0} \frac{\left.\operatorname{Vol}\left(\overline{S T_{\theta}(\alpha)}\right)^{\mathbb{R}} \cap B_{\epsilon}(0)\right)}{\left.\operatorname{Vol}\left(\overline{S T_{\theta}(\beta)}\right)^{\mathbb{R}} \cap B_{\epsilon}(0)\right)}=0 .
$$

We have the following volume properties analogous to those in [9].

Proposition 6.4. Let $\alpha, \beta \subset R^{n}$ be definable cones at $0 \in R^{n}$. Suppose that $\operatorname{dim} \alpha<$ $\operatorname{dim} \beta$. Then, for $\theta \in \Phi$,

$$
\lim _{\epsilon \rightarrow 0} \frac{\left.\operatorname{Vol}\left(\overline{S T_{\theta}(\alpha)}\right)^{\mathbb{R}} \cap B_{\epsilon}(0)\right)}{\left.\operatorname{Vol}\left(\overline{S T_{\theta}(\beta)}\right)^{\mathbb{R}} \cap B_{\epsilon}(0)\right)}=0 .
$$

Proof. Let $\gamma$ be a definable cone at $0 \in R^{n}$ of dimension $r$, and let $M$ be an $r$-dimensional linear subspace of $R^{n}$. Then the proposition follows easily from Lemma 6.3 and the fact that

$$
\operatorname{Vol}\left(\overline{S T_{\theta}(\gamma)}{ }^{\mathbb{R}} \cap B_{\epsilon}(0)\right) \approx \operatorname{Vol}\left(\overline{S T_{\theta}(M)}{ }^{\mathbb{R}} \cap B_{\epsilon}(0)\right)
$$

for $\theta \in \Phi$.

Let us show (6.1). We may assume that $\gamma$ is equidimensional. Then there exist a finite partition of $\gamma$ into $r$-dimensional definable cones $\gamma_{1}, \cdots, \gamma_{s}$ with $0 \in R^{n}$ as a vertex, and $r$-dimensional linear subspaces $M_{1}, \cdots, M_{s}$ of $R^{n}$ such that for each orthogonal projection $\Pi_{i}: R^{n} \rightarrow M_{i}, 1 \leq i \leq s, \gamma_{i}$ is expressed as the graph of a definable map from $\Pi_{i}\left(\gamma_{i}\right) \subset M_{i}$ and the diameter of $S T_{\theta}(\gamma) \cap \Pi_{i}^{-1}(u), u \in \Pi_{i}\left(\gamma_{i}\right)$, is less than or equal to $4 \theta$. Then we can see that

$$
\operatorname{Vol}\left(\overline{S T_{\theta}(\gamma)}{ }^{\mathbb{R}} \cap B_{\epsilon}(0)\right) \precsim \operatorname{Vol}\left({\overline{S T_{4 \theta}(M)}}^{\mathbb{R}} \cap B_{\epsilon}(0)\right)
$$


On the other hand, we may assume that one of $\Pi_{i}\left(\gamma_{i}\right)$ 's is a closed cone in $M_{i}$, taking a finite subdivision of $\gamma_{i}$ 's if necessary. Then we can see that $M_{i}$ is covered with a finite number of $r$-dimensional closed cones of the same size as $\Pi_{i}\left(\gamma_{i}\right)$. It follows that

$\left.\left.\operatorname{Vol}\left(\overline{S T_{\theta}\left(M_{i}\right)}\right)^{\mathbb{R}} \cap B_{\epsilon}(0)\right) \precsim \operatorname{Vol}\left(\overline{S T_{\theta}\left(M_{i}\right) \cap \Pi_{i}^{-1}\left(\Pi_{i}\left(\gamma_{i}\right)\right.}\right)^{\mathbb{R}} \cap B_{\epsilon}(0)\right) \precsim \operatorname{Vol}\left(\overline{S T_{\theta}\left(\gamma_{i}\right)}{ }^{\mathbb{R}} \cap B_{\epsilon}(0)\right)$.

Therefore we have

$$
\left.\left.\operatorname{Vol}\left(\overline{S T_{\theta}(\gamma)}\right)^{\mathbb{R}} \cap B_{\epsilon}(0)\right) \succsim \operatorname{Vol}\left(\overline{S T_{\theta}(M)}\right)^{\mathbb{R}} \cap B_{\epsilon}(0)\right)
$$

Then (6.1) follows from (6.2), (6.3) and Lemma 6.2.

The next lemma follows in the same way as Lemma 7.3 in [9]:

Lemma 6.5. Let $A \subset R^{n}$ be a definable set-germ at $0 \in R^{n}$ such that $0 \in \bar{A}$. Then we have $\operatorname{dim} L D(A) \leq \operatorname{dim} A$.

Proposition 6.6. Let $A, B$ be set-germs at 0 in $R^{n}$ such that $0 \in \bar{A} \cap \bar{B}$. Suppose that $A$ and $B$ are $S T$-equivalent. Then there is $\theta_{1} \in \Phi$ such that

$$
\operatorname{Vol}\left(\overline{S T_{\theta}(A)}{ }^{\mathbb{R}} \cap B_{\epsilon}(0)\right) \approx \operatorname{Vol}\left(\overline{S T_{\theta}(B)} \mathbb{R}^{\mathbb{R}} \cap B_{\epsilon}(0)\right)
$$

for any $\theta \in \Phi$ with $\theta \geq \theta_{1}$.

Proof. Let $\theta_{3}, \theta_{4} \in \Phi$ such that $A \subset S T_{\frac{\theta_{3}}{2}}(B), B \subset S T_{\frac{\theta_{4}}{2}}(A)$. Take $\theta_{1} \in \Phi$ so that $\theta_{1}(t) \geq 2 \max \left(\theta_{3}(2 t), \theta_{4}(2 t)\right)$ for $0<t<1$.

Claim. For any $\theta \in \Phi$ with $\theta \geq \theta_{1}$, we have

$$
S T_{\frac{\theta}{2}}(A) \subset S T_{\frac{\theta}{2}}\left(S T_{\frac{\theta_{3}}{2}}(B)\right) \subset S T_{2 \theta}(B) \subset S T_{4 \theta}(A)
$$

as germs at $0 \in R^{n}$.

Proof. To see the second inclusion, let $x \in S T_{\frac{\theta}{2}}\left(S T_{\frac{\theta_{3}}{2}}(B)\right)$ with $\theta(\|x\|) \leq 1$. Then there exists $y \in R^{n}$ such that $d(y, B) \leq \frac{\theta_{3}}{2}(\|y\|)\|y\|$ and $d(x, y) \leq \theta(\|x\|)\|x\|$. We also have $\|y\| \leq\|x\|+\theta(\|x\|)\|x\| \leq 2\|x\|$. Take $z \in B$ such that $d(y, z) \leq \theta_{3}(\|y\|)\|y\|$. Then

$$
\begin{aligned}
d(x, z) & \leq d(x, y)+d(y, z) \\
& \leq \theta(\|x\|)\|x\|+\theta_{3}(\|y\|)\|y\| \\
& \leq \theta(\|x\|)\|x\|+\theta_{3}(2\|x\|) 2\|x\| \\
& \leq \theta(\|x\|)\|x\|+\theta(\|x\|)\|x\|=2 \theta(\|x\|)\|x\| .
\end{aligned}
$$

This implies $x \in S T_{2 \theta}(B)$, and hence $S T_{\frac{\theta}{2}}\left(S T_{\frac{\theta_{3}}{2}}(B)\right) \subset S T_{2 \theta}(B)$.

Let $x \in S T_{2 \theta}\left(S T_{\frac{\theta_{4}}{2}}(A)\right)$ with $\theta(\|x\|) \leq \frac{1}{3}$. Similarly as above, we can show

$$
S T_{2 \theta}(B) \subset S T_{2 \theta}\left(S T_{\frac{\theta_{4}}{2}}(A)\right) \subset S T_{4 \theta}(A) .
$$

The statement of the proposition follows from this claim and Lemma 6.2.

The following corollary is an obvious consequence of Theorem 4.14, Lemma 6.5 and Propositions 6.4 and 6.6 . 
Corollary 6.7. Let $\alpha \subset R^{n}$ be a definable set-germ at $0 \in R^{n}$ such that $0 \in \bar{\alpha}$, and let $\beta \subset R^{n}$ be a definable cone at $0 \in R^{n}$. Suppose that $\operatorname{dim} \alpha<\operatorname{dim} \beta$. Then there is $\theta_{1} \in \Phi$ such that

$$
\lim _{\epsilon \rightarrow 0} \frac{\left.\operatorname{Vol}\left(\overline{S T_{\theta}(\alpha)}\right)^{\mathbb{R}} \cap B_{\epsilon}(0)\right)}{\left.\operatorname{Vol}\left(\overline{S T_{\theta}(\beta)}\right)^{\mathbb{R}} \cap B_{\epsilon}(0)\right)}=0
$$

for any $\theta \in \Phi$ with $\theta \geq \theta_{1}$.

We next describe a key lemma needed for proving our main theorem.

Lemma 6.8. Let $h:\left(R^{n}, 0\right) \rightarrow\left(R^{n}, 0\right)$ be a bi-Lipschitz homeomorphism, let $E \subset R^{n}$ be a definable set-germ at $0 \in R^{n}$ such that $0 \in \bar{E}$, and let $F:=h(E)$. Suppose that $F$ and $L D(F)$ are $S T$-equivalent and $L D(F)$ is definable. Then we have $\operatorname{dim} L D(F) \leq \operatorname{dim} E$.

Proof. Since $h:\left(R^{n}, 0\right) \rightarrow\left(R^{n}, 0\right)$ is a bi-Lipschitz homeomorphism and $R^{n}$ is dense in $\mathbb{R}^{n}, h$ has a natural extension to a bi-Lipschiz homeomorphism $\bar{h}:\left(\mathbb{R}^{n}, 0\right) \rightarrow\left(\mathbb{R}^{n}, 0\right)$. Then we have

$$
\left.{\overline{h\left(S T_{\theta}(E)\right)}}^{\mathbb{R}}=\bar{h}\left(\overline{S T_{\theta}(E)}\right)^{\mathbb{R}}\right) \text { for } \theta \in \Phi .
$$

Therefore it follows from Lemmas 4.8 and 6.2 that

$$
\left.\left.\operatorname{Vol}\left(\overline{S T_{\theta}(F)}\right)^{\mathbb{R}} \cap B_{\epsilon}(0)\right) \approx \operatorname{Vol}\left(\overline{S T_{\theta}(E)}\right)^{\mathbb{R}} \cap B_{\epsilon}(0)\right) .
$$

On the other hand, $F$ and $L D(F)$ are $S T$-equivalent. By Proposition 6.6, there is $\theta_{1} \in \Phi$ such that

$$
\left.\operatorname{Vol}\left({\overline{S T_{\theta}(F)}}^{\mathbb{R}} \cap B_{\epsilon}(0)\right) \approx \operatorname{Vol}\left(\overline{S T_{\theta}(L D(F)}\right)^{\mathbb{R}} \cap B_{\epsilon}(0)\right)
$$

for any $\theta \in \Phi$ with $\theta \geq \theta_{1}$.

By (6.4) and (6.5), we have

$$
1 \approx \frac{\left.\operatorname{Vol}\left(\overline{S T_{\theta}(F)}\right)^{\mathbb{R}} \cap B_{\epsilon}(0)\right)}{\left.\operatorname{Vol}\left(\overline{S T_{\theta}(L D(F)}\right)^{\mathbb{R}} \cap B_{\epsilon}(0)\right)} \approx \frac{\left.\operatorname{Vol}\left(\overline{S T_{\theta}(E)}\right)^{\mathbb{R}} \cap B_{\epsilon}(0)\right)}{\left.\operatorname{Vol}\left(\overline{S T_{\theta}(L D(F)}\right)^{\mathbb{R}} \cap B_{\epsilon}(0)\right)}
$$

for any $\theta \in \Phi$ with $\theta \geq \theta_{1}$. Assume that $\operatorname{dim} L D(F)>\operatorname{dim} E$. Then, by Corollary 6.7, the RHS ratio in (6.6) tends to 0 as $\epsilon \rightarrow 0$, if $\theta$ is sufficiently big. This is a contradiction. Thus we have $\operatorname{dim} L D(F) \leq \operatorname{dim} E$.

Let us show Proposition 6.1. The sets $A$ and $h(A)$ are assumed definable. Therefore, by Lemma 5.7, $L D(h(A))=L D(h(L D(A)))$ is definable, and it follows from Theorem 4.14 that $L D(A)$ is $S T$-equivalent to $A$. Then, by Proposition 4.9, $h(L D(A))$ is $S T$-equivalent to $h(A)$. In addition, it follows from the definability of $h(A)$, that $h(A)$ is $S T$-equivalent to $L D(h(A))=L D((h(L D(A)))$. Since the $S T$-equivalence is an equivalence relation, $h(L D(A))$ is $S T$-equivalent to $L D(h(L D(A)))$. Thus the proposition follows from Lemma 6.8 with $E=L D(A)$ and $F=h(L D(A))$, since $\operatorname{dim} h(L D(A))=\operatorname{dim} L D(A)$.

This completes the proof of our main theorem. 


\section{General Real Closed field CASe}

In this section we formulate and prove our main theorem for an arbitrary real closed field. Let $R$ denote a real closed field with an o-minimal structure and consider the topology on $R$ given by the open intervals of $R$, analogous to that on $\mathbb{R}$. We have already proved our main theorem for an arbitrary Archimedean real closed field. An example of a non-Archimedean real closed field is the field of Puiseux series, where a Puiseux series is a power series of the form $\sum_{i=k}^{\infty} a_{i} t^{i / p}$ for $a_{i} \in \mathbb{R}, p>0 \in \mathbb{N}$ and $k \in \mathbb{Z}$ such that $\sum_{i=\max (0, k)}^{\infty} a_{i} t^{i}$ is a formal (convergent) power series in one variable $t$. One reason for why we consider problems on a general real closed field $R$, is that some problems on $\mathbb{R}$ are solved by replacing $\mathbb{R}$ with $R$. Actually, the Hilbert 17 th problem is a famous illustration of this.

In order to treat our main theorem for $R$, we need to modify the previous definitions. Let $A$ and $B$ always denote subsets of $R^{n}$. Set

$$
\operatorname{dist}(A, B)=\{t \geq 0 \in R \mid \forall a \in A \forall b \in B, t \leq\|a-b\|\} .
$$

Here for $\left(x_{1}, \cdots, x_{n}\right) \in R^{n}$ we define $\left\|\left(x_{1}, \cdots, x_{n}\right)\right\|$ to be $\max _{i=1, \cdots, n}\left|x_{i}\right|$. Then $\operatorname{dist}(A, B)$ is a closed connected subset of $R$. In case $R=\mathbb{R}$, it is basically the closed interval with ends 0 and the usual distance between $A$ and $B$. In the general case if $A$ and $B$ are definable, $\operatorname{dist}(A, B)$ is also a closed interval with ends 0 and a number in $R$. (By abuse of notation $\operatorname{dist}(A, B)$ denotes also the right end number in the definable case.) However, for general subsets this is not the case, even if $R$ is Archimedean. Nevertheless in the Archimedean case we may understand $\operatorname{dist}(A, B)$ as a real number. For a general $R$ we do not know such an extension field, and therefore we have to define $\operatorname{dist}(A, B)$ to be a subset of $R$. For subsets $X$ and $Y$ of $R, X \leq Y$ means by definition that $\forall x \in X \exists y \in Y x \leq y$, and $X+Y$ denotes the set $\{x+y \mid x \in X, y \in Y\}$.

Let $D(A)$ denote the subset of $S^{n-1}=\left\{x \in R^{n} \mid\|x\|=1\right\}$ consisting of points $a$ such that $\forall \epsilon>0 \in R \forall \delta>0 \in R \exists x \in A-\{0\}$ with $\|x\| \leq \delta$, $\operatorname{dist}\left(a, \frac{x}{\|x\|}\right)<\epsilon$. In the Archimedean case, $D(A)$ coincides with that in Definition 2.2. It may be empty for general $A$, but if $A$ is definable then it is definable and not empty. We define $L D(A)$ in the same way as before, it is definable if $A$ is definable.

In the sense of the above notions of distance and direction set, we have the following:

Theorem 7.1. Let $A, B \subset R^{n}$ be definable set-germs at $0 \in R^{n}$ such that $0 \in \bar{A} \cap \bar{B}$, and let $h:\left(R^{n}, 0\right) \rightarrow\left(R^{n}, 0\right)$ be a bi-Lipschitz homeomorphism. Suppose that $h(A), h(B)$ are also definable. Then we have

$$
\operatorname{dim}(D(h(A)) \cap D(h(B)))=\operatorname{dim}(D(A) \cap D(B)) .
$$

Let us show Theorem 7.1. Define $\Phi$ as in the Archimedean case. Let $A \subset R^{n}$ such that $0 \in \bar{A}$. For $\theta \in \Phi$, we define the sea-tangle neighbourhood of $A$ by

$$
S T_{\theta}(A):=\left\{x \in R^{n} \mid \operatorname{dist}(x, A) \leq \theta(\|x\|)\|x\|\right\} .
$$

If $A$ is definable, so is $S T_{\theta}(A)$. If $R$ is Archimedean, $S T_{\theta}(A)$ coincides with that in Definition 4.4, $S T$-equivalence is also defined as before. 
Using the notions of $\Phi$, sea-tangle neighbourhood and condition $(S S P)$ as above, Lemma 4.8, Proposition 4.9, Lemma 4.10, Corollary 4.11, Proposition 4.12, Proposition 4.13, Theorem 4.14, Corollary 4.15, Theorem 4.16, Lemma 5.6, Lemma 5.7 and Proposition 5.8 are all proved for $R$, in the same way as before by replacing sequences with filters. There are no essential modifications until $\S 5$, because those arguments work in the family of definable sets and their images under bi-Lipschitz homeomorphisms. However we need to modify $\S 6$, in particular, the volume arguments.

For a subset $A \subset R^{n}$ we define $\operatorname{dim} A$ to be the dimension of the topological space $A$ as in the dimension theory (see [7]). There are no problems with this definition because $\operatorname{dim} A=$ $\operatorname{dim} h(A)$ for a homeomorphism $h$ of $R^{n}$, if $A \subset B$ then $\operatorname{dim} A \leq \operatorname{dim} B$, and if $A$ is definable then $\operatorname{dim} A$ coincides with the largest integer $k$, such that there exists a definable imbedding of $R^{k}$ into $A$. Then Theorem 7.1 follows from Proposition 6.1 for $R$ as in the Archimedean case. We will refer to Proposition 6.1 for $R$ as to the Generalised Proposition 6.1. If the germ of $A$ at $0 \in R^{n}$ in the statement of the Generalised Proposition 6.1 is of dimension $n$, then $\operatorname{dim} h(L D(A))=\operatorname{dim} L D(A)=n$ and the Generalised Proposition 6.1 holds true. Therefore we consider only subsets of $\left\{\left(x_{1}, \cdots, x_{n}\right)=\left(x^{\prime}, x_{n}\right) \in R^{n} \mid\left\|x^{\prime}\right\| \leq\right.$ $\left.c x_{n}, x_{n} \geq 0\right\}, c>0 \in R$, of dimension less than $n$. For each $\epsilon \geq 0 \in R$, set

$$
A_{\epsilon}:=\left\{x^{\prime} \in R^{n-1} \mid\left(x^{\prime}, \epsilon\right) \in A\right\} .
$$

Then we can change the previous definition of $S T_{\theta}(A)$ to

$$
\left\{\left(x^{\prime}, x_{n}\right) \in R^{n} \mid x_{n} \geq 0, \operatorname{dist}\left(x^{\prime}, A_{x_{n}}\right) \leq \theta\left(x_{n}\right) x_{n}\right\}
$$

which is easier to calculate.

The problem is how to define the volume of $A$. We do not have any good definition of volume in the general case. However the following definition is sufficient for our purpose. Assume that $A$ is bounded and definable.

In the following we will define $\operatorname{Vol} A$. For the simplest case of an open cube $O=$ $\left(a_{1}, b_{1}\right) \times \cdots \times\left(a_{n}, b_{n}\right)$ in $R^{n}$ with $a_{i} \leq b_{i} \in R$, let $V o l O$ be the expected product $\left(b_{1}-a_{1}\right) \cdots\left(b_{n}-a_{n}\right)$. Next consider the case where $A$ is the following set:

$$
\left\{\left(x_{1}, \ldots, x_{n}\right) \in R^{n} \mid a_{1}<x_{1}<b_{1}, a_{2}<x_{2}<\phi_{2}\left(x_{1}\right), \ldots, a_{n}<x_{n}<\phi_{n}\left(x_{1}, \ldots, x_{n-1}\right)\right\}
$$

for $a_{1}, \cdots, a_{n}, b_{1} \in R$ and bounded definable $C^{0}$ functions $\phi_{2}$ on $\left(a_{1}, b_{1}\right), \cdots, \phi_{n}$ on

$$
\begin{aligned}
& \left\{\left(x_{1}, \cdots, x_{n-1}\right) \in R^{n-1} \mid\right. \\
& \left.a_{1}<x_{1}<b_{1}, a_{2}<x_{2}<\phi_{2}\left(x_{1}\right), \cdots, a_{n-1}<x_{n-1}<\phi_{n-1}\left(x_{1}, \cdots, x_{n-2}\right)\right\} .
\end{aligned}
$$

We write $A=A_{a_{1}, \ldots, a_{n}, b_{1}, \phi_{2}, \ldots, \phi_{n}}$. For each $k=1,2, \cdots$, let $\operatorname{Vol}_{k} A$ denote the maximal number of $\sum_{i} \mathrm{VolO}_{i}$ where $\left\{O_{i}\right\}$ runs over all $k$ disjoint open cubes contained in $A$ (they exist since $A$ is definable). Note that $\left\{\operatorname{Vol}_{k} A\right\}_{k=1,2, \ldots}$ is increasing. From now on we identify $\operatorname{Vol}_{k} A$ with $\left\{t \geq 0 \in R \mid t \leq \operatorname{Vol}_{k} A\right\}$. Set $V o l A:=\cup_{k} V o l_{k} A$, which is a convex subset of $\{t \geq 0 \in R\}$ and contains 0 .

Consider the set $A$ of the form

$$
\begin{aligned}
& \left\{\left(x_{1}, \ldots, x_{n}\right) \in R^{n} \mid\right. \\
& \left.a_{1}<x_{1}<b_{1}, \psi_{2}\left(x_{1}\right)<x_{2}<\phi_{2}\left(x_{1}\right), \ldots, \psi_{n}\left(x_{1}, \ldots, x_{n-1}\right)<x_{n}<\phi_{n}\left(x_{1}, \ldots, x_{n-1}\right)\right\}
\end{aligned}
$$


for $a_{1}, b_{1} \in R$ and bounded definable $C^{0}$ functions $\psi_{i}$ and $\phi_{i}$ on

$$
\left\{\left(x_{1}, \ldots, x_{i-1}\right) \in R^{i-1} \mid a_{1}<x_{1}<b_{1}, \ldots, \psi_{i-1}\left(x_{1}, \ldots, x_{i-2}\right)<x_{i-1}<\phi_{i-1}\left(x_{1}, \ldots, x_{i-2}\right)\right\}
$$

with $\psi_{i}<\phi_{i}, i=2, \cdots, n$. We call $A$ of cell form and write $A=A_{a_{1}, \psi_{2}, \ldots, b_{1}, \phi_{2}, \ldots}$. If we define $\operatorname{Vol} A$ for $A$ of cell form in the same way as above, then our arguments do not work. For example, let $\epsilon>0 \in R$ be smaller than any positive real number and set $A=A_{0, x, 1, \epsilon+x}$ in $R^{2}$. Then

$$
\operatorname{Vol} A=\left\{a \geq 0 \in R \mid \exists k \in \mathbb{N} a \leq k \epsilon^{2}\right\} .
$$

However we expect $\operatorname{Vol} A=[0, \epsilon]$, both from the context of the proofs in $\S 6$ and from the following arguments. Here we introduce an artificial different definition of volume. Choose $a_{2}, \cdots, a_{n} \in R$ so small that $a_{2}<\psi_{2}, \cdots, a_{n}<\psi_{n}$. Then

$$
A=A_{a_{1}, \ldots, a_{n}, b_{1}, \phi_{2}, \ldots, \phi_{n}}-\cup \overline{A_{a_{1}, \ldots, a_{n}, b_{1}, \rho_{2}, \ldots, \rho_{n}}},
$$

where $\left\{\rho_{2}, \ldots, \rho_{n}\right\}$ satisfy $\rho_{i}=\psi_{i}$ for one $i$ and $\rho_{j}=\phi_{j}$ for the other $j^{\prime} s$. For such distinct families $\left\{\rho_{i}\right\}$ and $\left\{\rho_{i}^{\prime}\right\}$ we have

$$
A_{a_{1}, \ldots, a_{n}, b_{1}, \rho_{2}, \ldots, \rho_{n}} \cap A_{a_{1}, \ldots, a_{n}, b_{1}, \rho_{2}^{\prime}, \ldots, \rho_{n}^{\prime}}=A_{a_{1}, \ldots, a_{n}, b_{1}, \min \left(\rho_{2}, \rho_{2}^{\prime}\right), \ldots, \min \left(\rho_{n}, \rho_{n}^{\prime}\right)} .
$$

If $R=\mathbb{R}$ then

$$
\operatorname{Vol} A=\sum p_{\xi_{2}, \ldots, \xi_{n}} \operatorname{Vol} A_{a_{1}, \ldots, a_{n}, b_{1}, \xi_{2}, \ldots, \xi_{n}}
$$

in the usual sense of volume for some integers $p_{\xi_{2}, \ldots, \xi_{n}}$ where $\left\{\xi_{2}, \cdots, \xi_{n}\right\}$ are so that for all $i, \xi_{i}=\psi_{i}$ or $\xi_{i}=\phi_{i}$. Here the $p_{\xi_{2}, \ldots, \xi_{n}}$ 's do not depend on the special choice of $\psi_{i}$ and $\phi_{i}$. For a general $R$, set

$$
\begin{aligned}
& V o l_{k} A:=\sum p_{\xi_{2}, \ldots, \xi_{n}} \operatorname{Vol}_{k} A_{a_{1}, \ldots, a_{n}, b_{1}, \xi_{2}, \ldots, \xi_{n}}, \\
& V o l A:=\cap_{l=1}^{\infty} \cup_{k=l}^{\infty} \operatorname{Vol}_{k} A,
\end{aligned}
$$

i.e., $\epsilon \geq 0 \in \operatorname{Vol} A$ if and only if $\forall l \in \mathbb{N} \exists k \geq l \in \mathbb{N} \epsilon \in \operatorname{Vol}_{k} A$.

Note that $\operatorname{Vol} A_{0, x, 1, \epsilon+x}=[0, \epsilon]$, and if $A$ is of cell form and is a disjoint union of a definable set of dimension less than $n$ and a finite number of definable sets $A_{i}$ of cell form, then $\operatorname{Vol} A=\sum_{i} \operatorname{Vol} A_{i}$.

Let $A$ be a general bounded definable set in $R^{n}$. Then, by the Cell Decomposition Theorem, $A$ is a disjoint union of a definable set of dimension less than $n$ and a finite number of definable sets $A_{i}$ of cell form. Set $\operatorname{Vol} A=\sum_{i} \operatorname{Vol} A_{i}$. Then $\operatorname{Vol} A$ does not depend on the choice of cell decomposition. If $B$ is open, bounded and definable and contains $\bar{A}$, then $\operatorname{Vol} A \varsubsetneqq \operatorname{Vol} B ; \operatorname{Vol} A=\operatorname{Volh}(A)$ for the map $h: R^{n} \ni\left(x_{1}, \ldots, x_{n}\right) \rightarrow$ $\left(x_{\tau(1)}, \ldots, x_{\tau(n)}\right) \in R^{n}$ where $\tau$ is a permutation of $\{1, \ldots, n\}$. For disjoint bounded definable sets $A_{1}$ and $A_{2}$ we have $\operatorname{Vol}\left(A_{1} \cup A_{2}\right)=\operatorname{Vol} A_{1}+\operatorname{Vol} A_{2}$.

This is the definition of volume of definable subsets of $R^{n}$.

For a definable subset $A$ of $R^{n}$ and for each $\epsilon \geq 0 \in R, V o l A_{\epsilon}$ is a subset of $R$. Note that $V o l A_{\epsilon}$ is calculated regarding $A_{\epsilon}$ as a subset of $R^{n-1}$. We are interested in the correspondence $[0, \infty) \ni \epsilon \rightarrow \operatorname{Vol}_{\epsilon} \subset R$. Let $V o l_{A}$ denote the correspondence. Let $f$ and $g$ be maps from $[0, \infty)$ to the power set $\mathfrak{B}(R)$. If there are $K>0$ and $\delta>0$ in $R$ such that

$$
f(\epsilon) \subset K g(\epsilon) \subset K^{2} f(\epsilon) \text { for } \quad \epsilon \in[0, \delta],
$$


we write $f \approx g$. By $\lim _{\epsilon \rightarrow 0} \frac{g(\epsilon)}{f(\epsilon)}=0$, we mean that $\forall \epsilon>0 \in R \exists \delta>0 \in R \forall \epsilon_{1} \epsilon$ $(0, \delta] g\left(\epsilon_{1}\right) \subset \epsilon f\left(\epsilon_{1}\right) \neq\{0\}$.

For subsets $X$ and $Y$ of $R^{n}, \operatorname{Vol}_{X} \approx \operatorname{Vol}_{Y}$ means that there are definable sets $A_{1}, A_{2}, B_{1}$ and $B_{2}$ in $R^{n}$ such that

$$
A_{1} \subset X \subset A_{2}, B_{1} \subset Y \subset B_{2} \text { and } \operatorname{Vol}_{A_{1}} \approx \operatorname{Vol}_{A_{2}} \approx V_{o l} l_{B_{1}} \approx \operatorname{Vol}_{B_{2}} .
$$

In the same way we define $\lim _{\epsilon \rightarrow 0} \frac{V o l_{X}(\epsilon)}{V o l_{Y}(\epsilon)}=0$.

Because of Lemma 6.2, we may expect $V o l_{S T_{c \theta}(X)} \approx V_{o l T_{\theta}(X)}$, for $X \subset R^{n}, \theta \in \Phi$ and $c>0 \in R$. However we do not know whether this is the case. We first prove:

Lemma 7.2. Let $A$ be a definable cone at $0 \in R^{n}$. Then

$$
\operatorname{Vol}_{S T_{c \theta}(A)} \approx \operatorname{Vol}_{S T_{\theta}(A)}
$$

for $\theta \in \Phi$ and $c>0 \in R$.

Proof. Assume $c>1$, and let $A$ be the cone with base $C \times\{1\} \subset R^{n-1} \times R$, where we assume that $C$ is closed. Since $C$ admits a finite stratification with definable $C^{1}$ manifolds, we only need to prove the lemma for the cones with vertex 0 and base each of the strata. In addition, as in $\S I I .1$ in [13], we can choose the stratification so that for each stratum $C_{1}$ of dimension $k$, there exist $n_{1}<\cdots<n_{k}$ in $\{1, \cdots, n\}$ (assume, for the simplicity of notation, that $\left.n_{i}=i, i=1, \cdots, k\right)$, such that the restriction to $\overline{C_{1}}$ (not only to $C_{1}$ ) of the projection $p: R^{n-1} \ni\left(x_{1}, \cdots, x_{n-1}\right) \rightarrow\left(x_{1}, \cdots, x_{k}\right) \in R^{k}$ is injective, i.e., $\overline{C_{1}}$ is the graph of some $C^{0}$ map $\alpha=\left(\alpha_{1}, \cdots, \alpha_{n-1-k}\right): p\left(\overline{C_{1}}\right) \rightarrow R^{n-1-k},\left.\alpha\right|_{p\left(C_{1}\right)}$ is of class $C^{1}$ and $\left\|\left.\operatorname{grad} \alpha_{i}\right|_{p\left(C_{1}\right)}\right\| \leq 1$ for $i=1, \cdots, n-1-k$.

What we want to prove is that there exists $K>0 \in R$ such that

$$
\operatorname{Vol}\left\{x^{\prime} \in R^{n-1} \mid \operatorname{dist}\left(x^{\prime}, C_{1}\right) \leq c c^{\prime}\right\} \leq K \operatorname{Vol}\left\{x^{\prime} \in R^{n-1} \mid \operatorname{dist}\left(x^{\prime}, C_{1}\right) \leq c^{\prime}\right\}
$$

for small $c^{\prime} \geq 0 \in R$. Proceeding by induction on $k$, we can reduce the problem to

$$
\begin{aligned}
& \operatorname{Vol}\left\{x^{\prime} \in p^{-1}\left(p\left(C_{1}\right)\right) \mid \operatorname{dist}\left(x^{\prime}, C_{1}\right) \leq c c^{\prime}\right\} \\
& \leq K \operatorname{Kol}\left\{x^{\prime} \in p^{-1}\left(p\left(C_{1}\right)\right) \mid \operatorname{dist}\left(x^{\prime}, C_{1}\right) \leq c^{\prime}\right\} .
\end{aligned}
$$

By the definition of volume

$$
\begin{aligned}
& \operatorname{Vol}\left\{x^{\prime} \in p^{-1}\left(p\left(C_{1}\right)\right) \mid \operatorname{dist}\left(x^{\prime}, C_{1}\right) \leq c c^{\prime}\right\} \\
& \leq \operatorname{Vol}\left\{x^{\prime} \in p^{-1}\left(p\left(C_{1}\right)\right) \mid \operatorname{dist}\left(x^{\prime}, p\left(C_{1}\right)\right) \leq c c^{\prime}\right\} .
\end{aligned}
$$

On the other hand, since $\left\|\left.\operatorname{grad} \alpha_{i}\right|_{p\left(C_{1}\right)}\right\| \leq 1$,

$$
\begin{aligned}
& \operatorname{Vol}\left\{x^{\prime} \in p^{-1}\left(p\left(C_{1}\right)\right) \mid \operatorname{dist}\left(x^{\prime}, p\left(C_{1}\right)\right) \leq c^{\prime}\right\} \\
& \leq 2^{n-1-k} \operatorname{Vol}\left\{x^{\prime} \in p^{-1}\left(p\left(C_{1}\right)\right) \mid \operatorname{dist}\left(x^{\prime}, C_{1}\right) \leq c^{\prime}\right\} .
\end{aligned}
$$

Thus we can replace $C_{1}$ in (7.1) with $p\left(C_{1}\right)$. Clearly (7.1) for $p\left(C_{1}\right)$ holds true for $K=$ $c^{n-1-k}$.

We generalise the above lemma as follows.

Lemma 7.2 Let $A$ be a definable set-germ at $0 \in R^{n}$. Then there exists $\theta_{1} \in \Phi$ such that

$$
V o l_{S T_{c \theta}(A)} \approx \operatorname{Vol}_{S T_{\theta}(A)}
$$


for $\theta \in \Phi$ and $c>0 \in R$ with $\theta \geq \theta_{1}$.

Proof. By Theorem $4.14 A$ is $S T$-equivalent to $L D(A)$. Therefore there exist $\theta_{2}, \theta_{3} \in \Phi$ such that

$$
L D(A) \subset S T_{\theta_{2}}(A) \text { and } A \subset S T_{\theta_{3}}(L D(A)) .
$$

Set $\theta_{1}=2 \max \left(\theta_{2}, \theta_{3}\right)$. Then, as in the proof of Proposition [6.6, we have

$$
S T_{\frac{\theta}{2}}(L D(A)) \subset S T_{\theta}(A) \subset S T_{2 \theta}(L D(A))
$$

for $\theta \in \Phi$ with $\theta \geq \theta_{1}$. Since $L D(A)$ is a definable cone with vertex $0 \in R^{n}$, by Lemma 7.2

Hence

$$
\operatorname{Vol}_{S T_{\frac{\theta}{2}}(L D(A))} \approx \operatorname{Vol}_{S T_{\frac{c \theta}{2}}(L D(A))} \approx \operatorname{Vol}_{S T_{2 c \theta}(L D(A))} \approx \operatorname{Vol}_{S T_{2 \theta}(L D(A))}
$$

$$
V o l_{S T_{c \theta}(A)} \approx \operatorname{Vol}_{S T_{\theta}(A)}
$$

By the above arguments the following lemma is clear; it corresponds to Lemma 6.3 ,

Lemma 7.3. Let $\alpha, \beta$ be linear subspaces of $R^{n-1}$ with $\operatorname{dim} \alpha<\operatorname{dim} \beta$. Let $\alpha_{1}$ and $\beta_{1}$ denote the cones in $R^{n}$ with vertex $0 \in R^{n}$ and bases $\left\{x^{\prime} \in \alpha \mid\left\|x^{\prime}\right\| \leq 1\right\} \times\{1\}$ and $\left\{x^{\prime} \in \beta \mid\left\|x^{\prime}\right\| \leq 1\right\} \times\{1\}$, respectively. Then, for $\theta \in \Phi$,

$$
\lim _{\epsilon \rightarrow 0} \frac{\operatorname{Vol}_{S T_{\theta}\left(\alpha_{1}\right)}(\epsilon)}{\operatorname{Vol}_{S T_{\theta}\left(\beta_{1}\right)}(\epsilon)}=0 .
$$

Using Lemmas 7.2 and 7.3 , we can show the following proposition in the same way as in the proof of Proposition 6.4,

Proposition 7.4. Let $\alpha, \beta \subset R^{n}$ be definable cones at $0 \in R^{n}$. Suppose that $\operatorname{dim} \alpha<$ $\operatorname{dim} \beta$. Then, for $\theta \in \Phi$,

$$
\lim _{\epsilon \rightarrow 0} \frac{V o l_{S T_{\theta}(\alpha)}(\epsilon)}{V o l_{S T_{\theta}(\beta)}(\epsilon)}=0 .
$$

The following lemma is clear.

Lemma 7.5. Let $A \subset R^{n}$ be a definable set-germ at $0 \in R^{n}$. Then we have $\operatorname{dim} L D(A) \leq$ $\operatorname{dim} A$.

We do not know whether Proposition 6.6 holds for general $R$. However, under some assumption, using Lemma 7.2 as in the proof of Proposition 6.6, we can prove the following.

Proposition 7.6. Let $A, B$ be set-germs at $0 \in R^{n}$. Suppose that $A$ and $B$ are $S T$ equivalent and $A$ is definable. Then there exists $\theta_{1} \in \Phi$ such that

$$
V o l_{S T_{\theta}(A)} \approx \operatorname{Vol}_{S T_{\theta}(B)}
$$

for any $\theta \in \Phi$ with $\theta \geq \theta_{1}$.

The following corollary is also clear. 
Corollary 7.7. Let $\alpha \subset R^{n}$ be a definable set-germ at $0 \in R^{n}$, and let $\beta \subset R^{n}$ be a definable cone at $0 \in R^{n}$. Suppose that $\operatorname{dim} \alpha<\operatorname{dim} \beta$. Then there $i s \theta_{1} \in \Phi$ such that

$$
\lim _{\epsilon \rightarrow 0} \frac{V o l_{S T_{\theta}(\alpha)}(\epsilon)}{\operatorname{Vol}_{S T_{\theta}(\beta)}(\epsilon)}=0
$$

for any $\theta \in \Phi$ with $\theta \geq \theta_{1}$.

We need to modify Lemma 6.8 as follows.

Lemma 7.8. Let $h:\left(R^{n}, 0\right) \rightarrow\left(R^{n}, 0\right)$ be a bi-Lipschitz homeomorphism, and let $A$ be a definable set-germ at $0 \in R^{n}$. Suppose that $h(A)$ is definable. Set $E=L D(A)$ and $F=h(E)$. Then $\operatorname{dim} L D(F) \leq \operatorname{dim} E$.

Proof. In the proof of Lemma 6.8, (6.4) was a consequence of Lemmas 4.8 and 6.2, For a general $R,(6.4)$ corresponds to

$$
\operatorname{Vol}_{S T_{\theta}(F)} \approx \operatorname{Vol}_{S T_{\theta}(E)} \quad \text { for } \quad \theta \in \Phi .
$$

If $F$ is definable, (7.2) follows from Lemmas 4.8 and 7.2. However $F$ is not necessarily definable or we do not know whether (7.2) holds for any $\theta$. We will find $\theta_{1} \in \Phi$ such that (7.2) holds for $\theta \in \Phi$ with $\theta \geq \theta_{1}$. Such a restriction does not yield any trouble in our proof.

Since $A$ and $E$ are $S T$-equivalent, by Proposition 7.6 there exists $\theta_{1} \in \Phi$ such that

$$
\operatorname{Vol}_{S T_{\theta}(A)} \approx \operatorname{Vol}_{S T_{\theta}(E)}
$$

for any $\theta \in \Phi$ with $\theta \geq \theta_{1}$. By the same reason as above and Lemma 5.6 we can assume that

$$
\operatorname{Vol}_{S T_{\theta}(h(A))} \approx \operatorname{Vol}_{S T_{\theta}(F)} .
$$

On the other hand, as in the proof of Lemma 6.8, by Lemmas 4.8 and 7.2 we have

$$
\operatorname{Vol}_{S T_{\theta}(A)} \approx \operatorname{Vol}_{S T_{\theta}(h(A))} .
$$

Hence (7.2) holds for $\theta \geq \theta_{1} \in \Phi$.

The other arguments in the proof of Lemma 6.8 continue to work.

The Generalised Proposition 6.1 and then Theorem 7.1 are proved in the same way as in $\S 6$.

\section{REFERENCES}

[1] S. Banach, Wstep do teorii funkcji rzeczywistych, Monografie matematyczne, Warszawa-Wroclaw, 1951, (in Polish).

[2] M. Coste, An introduction to o-minimal geometry, Dottorato di Ricerca in Matematica, Dip. Mat. Pisa. Instituti Editoriali e Poligrafici Internazionali, 2000.

[3] R. J. Daverman, Decompositions of manifolds, Pure and Applied Mathematics 124, Academic Press, 1986.

[4] L. van den Dries, Tame topology and o-minimal structures, LMS Lecture Notes Series 248, Cambridge University Press, 1997. 
[5] L. van den Dries and C. Miller, Geometric categories and o-minimal structures, Duke Math. Journal, 84 (1996), 497-540.

[6] H. Hironaka, Subanalytic sets, Number Theory, Algebraic Geometry and Commutative Algebra, in honor of Yasuo Akizuki, pp. 453-493, Kinokuniya, Tokyo, 1973.

[7] W. Hurewicz and H. Wallman, Dimension Theory, Princeton University Press, 1941.

[8] R. C. Kirby and L. C. Siebenmann, Foundational essays on topological manifolds, smoothings, and triangulations, Annals of Mathematics Studies 88, Princeton University Press, 1977.

[9] S. Koike and L. Paunescu, The directional dimension of subanalytic sets is invariant under biLipschitz homeomorphisms, Annales de l'Institut Fourier 59 (2009), 2445-2467.

[10] Ta Lê Loi, Lojasiewicz inequalities for sets definable in the structure $\mathbb{R}_{\text {exp }}$, Annales de l'Institut Fourier 45 (1995), 951-971.

[11] S. Lojasiewicz, Ensembles semi-analytiques. Inst. Hautes Etudes Sci. Lectute Note, 1967.

[12] C. P. Rourke and B. J. Sanderson, Introduction to piecewise-linear topology, Springer, 1977.

[13] M. Shiota, Geometry of subanalytic and semialgebraic sets, Progress in Mathematics 150, Birkhäuser, 1997.

[14] D. Sullivan, Hyperbolic geometry and homeomorphisms, Geometric topology, Proc. Georgia Topology Conf., Athens, Ga., 1977, Academic Press, 1979.

[15] A. J. Wilkie, Model completeness results for expansions of the ordered field of real numbers by restricted Pfaffian functions and the exponential functions, Jour. Amer. Math. Soc. 9 (1996), 10511094.

Department of Mathematics, Hyogo University of Teacher Education, Kato, Hyogo 673-1494, JAPAN

E-mail address: koike@hyogo-u.ac.jp

Department of Mathematics, University of Dalat, Dalat, Vietnam

E-mail address: taleloi@hotmail.com

School of Mathematics, University of Sydney, Sydney, NSW, 2006, Australia

E-mail address: laurentiu.paunescu@sydney.edu.au

Graduate School of Mathematics, Nagoya University, Furo-Cho, Chigusa-Ku, Nagoya 464-8602, JAPAN

E-mail address: shiota@math.nagoya-u.ac.jp 